\title{
Questions éthiques et cliniques posées par l'adoption de l'initiative sur l'internement à vie des délinquants particulièrement dangereux par le peuple suisse*
}

\section{Bruno Graviera, René Raggenbass ${ }^{b}$, Jacques Gasserc}

a professeur associé, médecin chef, Service de Médecine et Psychiatrie Pénitentiaires, Lausanne

b psychiatre-psychothérapeute FMH, Martigny

c professeur associé, médecin chef, Département de Psychiatrie, Lausanne
Correspondance:

Prof. Bruno Gravier SMPP

Clinique psychiatrique

universitaire

Site de Cery

CH-1008 Prilly

Bruno.Gravier@chuv.ch

\section{Résumé}

Une initiative populaire se concluant par un vote national a conduit le peuple suisse à demander une modification de sa constitution pour permettre d'interner définitivement certains délinquants particulièrement dangereux. Une fois la décision prise, aucune véritable réévaluation de la situation ne pourra être effectuée. Cette décision se basera principalement sur l'expertise psychiatrique qui devra se prononcer sur le caractère inamendable ou inaccessible à tout traitement de tels sujets.

Cet article présente l'historique de la démarche populaire qui a conduit à ce vote et la manière dont l'Etat suisse a rendu compte de cette votation en tentant de l'inscrire dans son code pénal.

Pourtant le texte voté se présentait d'une manière telle qu'il rendait impossible son inscription autant dans un discours juridique que dans un discours psychiatrique. Il confronte maintenant les praticiens à des questions éthiques majeures.

Doit-on accepter de s'inscrire dans un tel processus pénal? Peut-on valider par une expertise un pronostic qui engage la vie entière d'une personne alors que l'on connaît le caractère incertain et donc discutable des approches prédictives? Comment tout un Etat de droit a-t-il pu laisser se développer un tel leurre quant à la place de la psychiatrie dans la cité?

\section{Introduction}

La société témoigne d'une préoccupation grandissante pour la criminalité sexuelle. En premier lieu, en raison de la prise de conscience croissante de la réalité de ce phénomène chez les enfants et les adolescents. On s'inquiète aussi de l'ampleur de cette criminalité à la faveur du développement et de l'utilisation d'Internet. Les sites de rencontres et d'échanges y fleurissent, les réseaux semblent s'y développer. Ce climat contribue à une inquiétude diffuse face à un danger difficile à cerner.
Cette prise de conscience concerne également la gravité des conséquences immédiates et à long terme, en particulier psychiques, des agressions sexuelles sur les victimes.

De plus, le caractère récidivant de ces infractions, le sentiment de leur étrange proximité car elles peuvent soudain frapper au cœur de la famille ou du groupe social - transmettent un sentiment d'urgence à agir.

Enfin, le sentiment de désarroi qui émerge des discours sur les prises en charge, qu'elles soient pénales ou thérapeutiques ne pouvait que légitimer la violence de la réaction populaire dans la mesure où elle était sollicitée par un vote comme le permet le système démocratique suisse. En effet, l'insuffisance de moyens et de thérapeutes capables de prendre en charge ces sujets mais encore l'inadéquation des sanctions et le décalage entre la réalité judiciaire (peines légères dans la grande majorité des cas) et les représentations d'horreur dans la population, englobant toutes les délinquances sexuelles, viennent ponctuer les discours sécuritaires et renforcer l'inquiétude.

C'est dans ce contexte qu'a été adopté par le peuple suisse un texte constitutionnel permettant d'interner à vie, sans possibilité de réexamen ou de sortie, des criminels réputés particulièrement dangereux.

\section{Historique de l'initiative}

En Suisse, une modification constitutionnelle peut être portée au vote populaire après qu'une initiative signée par 100000 citoyens soit déposée devant le Parlement et le Conseil Fédéral. Celle-ci a recueilli 194390 signatures.

Il faut dire que ces signatures partaient du cœur de l'émotion la plus vive et la plus respectable puisque l'initiative était portée principalement par les parents et proches d'une victime sauvagement assassinée par un tueur en série dont la sinistre trajectoire a marqué les esprits depuis la fin des années 1980 .

La rumeur populaire véhiculait en outre, à torts, le fait que ce criminel bénéficiait de congés 
et avait été vu par les uns et les autres dans l'une ou l'autre ville. Quelques mois auparavant, l'examen de sa mise en liberté conditionnelle, obligatoire après 15 ans, en Suisse pour les condamnés à perpétuité avait aussi suscité l'inquiétude d'une possible sortie (cet examen a motivé une expertise qui a conclu à la nécessité de repousser le prochain examen de 10 ans).

Un autre crime terrible avait secoué l'opinion publique en 1993 puisqu'il était le fait d'un détenu déjà condamné pour des faits particulièrement graves et qui avait récidivé alors qu'il bénéficiait de congés quelques années après le début de sa peine dans des conditions d'octroi discutables et prématurées.

Le caractère démagogique et irréaliste d'une telle proposition rendait, en première lecture, son approbation impensable. En effet, le texte proposé obligeait la Suisse s'il était admis, à se mettre en rupture du reste de l'Europe par la contradiction avec les principes fondamentaux de la Convention Européenne des Droits de l'Homme. Son caractère extrémiste ne pouvait, de plus, qu'être rejeté par un pays traditionnellement enclin à la modération pénale et dont le code et les pratiques pénales sont principalement orientés vers la resocialisation et le caractère didactique de la sanction.

De plus, un nouveau code pénal venait d'être adopté en 2002 dont la mise en œuvre, initialement prévue pour 2006, allait renforcer certaines dimensions répressives du droit des mesures. Celles-ci jusqu'à présent permettant de garder sous main de justice des auteurs de crimes ou délits souffrant de troubles psychiques, de toxicomanie ou de comportements invétérés (délinquance d'habitude). La mesure étant une privation de liberté renouvelable d'année en année, et donc potentiellement indéterminée, venant en alternative à une peine de prison, celle-ci étant alors suspendue dans la majorité des cas. La mesure était donc, jusqu'à présent considérée dans l'esprit du législateur comme une stimulation au traitement et une alternative humaniste à l'enfermement carcéral.

Ces dernières années, il faut bien le dire, certaines mesures (internement au sens de l'article 43 CPS ch1 al 2) ont progressivement affirmé leur caractère sécuritaire, et sont maintenant comprises comme les réponses les plus répressives mises à la disposition des autorités de jugement.

Malgré le succès de ce premier recueil de signature, le vote sur l'initiative s'est préparé dans la quasi certitude des différents protagonistes étatiques (partis, députés, juristes, etc.) que celleci n'avait aucune chance d'être adoptée.
Ethische und klinische Fragen, hervorgerufen durch die Annahme der Verwahrungsinitiative durch das Schweizer Volk

Die Volksinitiative, die in eine nationalen Abstimmung mündete, führte zu einer Änderung der Bundesverfassung, damit bestimmte besonders gefährliche Täter auf Lebenszeit verwahrt werden können. Ist ein Urteil dieser Art einmal gefällt, kann keine wirkliche Reevaluation der Situation mehr vorgenommen werden. Die Entscheidung basiert hauptsächlich auf einem psychiatrischen Gutachten, das sich darüber äussern soll, wie unabänderlich oder wie unempfänglich für jegliche Behandlung der Charakter des beurteilten Menschen ist. Der Artikel zeichnet die Entstehungsgeschichte der Verwahrungsinitiative, die zu der genannten Abstimmung geführt hat, nach und stellt dar, wie der schweizerische Staat das Abstimmungsresultat in sein Strafgesetzbuch einzuführen versuchte.

Der Abstimmungstext ist jedoch in einer Weise abgefasst, die eine direkte Umsetzung sowohl unter psychiatrischen als auch unter juristischen Gesichtspunkten unmöglich machte. Momentan sind die Praktiker mit grösseren ethischen Fragen konfrontiert.

Soll man es akzeptieren, in einen solchen Strafprozess einbezogen zu werden? Kann man durch ein Gutachten einer Prognose über das gesamte Leben eines Menschen Geltung verschaffen, wenn man sich der unsicheren und deshalb diskutierbaren Auslegung von prädiktiven Betrachtungsweisen bewusst ist? Wie konnte sich ein Rechtsstaat in ein solches Gauklerspiel einbinden lassen, was die Psychiatrie in diesem Kontext betrifft?

En effet, elle avait unanimement suscité l'opposition du gouvernement fédéral et un rejet par les deux chambres de représentants élus. Plusieurs négociations avaient eu lieu entre partisans de l'initiative et représentants de l'Etat pour essayer de trouver un compromis dans le cadre de l'élaboration du nouveau code pénal et éviter ce vote. L'unanimité était telle dans la plupart des milieux concernés pour estimer irréaliste une 
telle proposition que tout n'a peut-être pas été fait, autant en termes de négociation que d'explication, pour se préoccuper d'une telle menace.

Pour certains [1], l'initiative s'est développée au moment même où la prise de conscience des autorités avait permis de développer de meilleurs outils pour suivre les condamnés et arriver à une meilleure appréciation des capacités de récidive des détenus: commissions d'évaluation de la dangerosité, expertises en cours d'exécution de peine, prises en charge spécialisées, etc. Cet effort méritoire semblant lui aussi relativiser la portée du vote. Il n'a cependant pas été entendu, probablement parce que ces dispositions, dont les effets ne peuvent se faire sentir que sur la durée, n'avaient pas encore suffisamment montré leurs résultats.

Personne n'avait véritablement pris la mesure de la lame de fond que les propos condensés dans les propositions simples et «évidentes» du texte de l'initiative ne pouvaient manquer de susciter.

Elle a donc été largement acceptée, contre les recommandations de la classe politique quasi unanime, à quelques exceptions près, retrouvées dans les partis populistes et contre les recommandations des milieux spécialisés.

Le texte adopté a donc la force de l'émotion, la simplicité de la réponse et la puissance des idées reçues face à l'horreur. La complexité du droit, la réalité des pratiques et les limites des uns et des autres ne pouvaient qu'être balayées à partir du moment où le texte était soumis.

Cette initiative constitutionnelle a été adoptée par 56\% des votants et la majorité des cantons en février 2004. En voici le texte:

«Si un délinquant sexuel ou violent est qualifié d'extrêmement dangereux et non amendable dans les expertises nécessaires au jugement, il est interné à vie en raison du risque élevé de récidive. Toute mise en liberté anticipée et tout congé sont exclus.

De nouvelles expertises ne sont effectuées que si de nouvelles connaissances scientifiques permettent d'établir que le délinquant peut être amendé et qu'il ne présente dès lors plus de danger pour la collectivité. L'autorité qui prononce la levée de l'internement au vu de ces expertises est responsable en cas de récidive.

Toute expertise concernant le délinquant est établie par au moins deux experts indépendants qui prennent en considération tous les éléments pertinents. (Art. 123a CST)»

\section{Du vote à la loi: inscrire l'inquiétude populaire en termes législatifs}

C'est dire à quel point de tels votes, expression du ressenti populaire, se fondent principalement sur de l'irrationnel et sur une rhétorique de la peur qui ne peut qu'échapper à toute cohérence et surtout à toute inscription dans une pensée ju- ridique et pénale. C'est à cette réalité que sont maintenant confrontés juristes et politiques, pris en tenaille entre la nécessité de tenir compte du vote du peuple souverain et les impasses et implications désastreuses d'un tel texte.

On peut estimer que les citoyens n'ont pas véritablement réalisé sur quoi ils votaient tant les enjeux étaient difficiles à décrypter, tant les discours de ceux qui appelaient à accepter l'initiative avaient tendance à considérer que ceux qui refusaient l'initiative semblaient s'ériger en défenseurs des auteurs d'agression sexuelle.

Refuser une telle initiative pouvait ainsi vouloir dire, pour beaucoup de citoyens, qu'on allait laisser sortir des délinquants sexuels, et notamment prendre le risque de retrouver dehors, du jour au lendemain, les grands «prédateurs» dont le crime avait été à l'origine de la démarche de l'initiative. C'était de cette manière qu'était comprise la question.

Finalement le peuple s'est découvert législateur et un législateur finalement plus féroce encore que ceux qui étaient à l'origine de cette proposition.

Car on peut aussi penser que si la question avait concerné l'ensemble des délinquants sexuels quelle que soit la gravité du délit, la réponse aurait été peu différente.

Bien sûr, un tel vote populaire est venu souligner dans un pays en quête de repères la défiance majeure vis-à-vis des juges et des psychiatres amalgamés dans une représentation de l'Etat, incapables d'assurer la sécurité de ses citoyens, de répondre à leurs attentes et coupables de laxisme vis-à-vis de ces criminels. On peut donc mesurer à quel point l'écart s'est creusé et les attentes se sont faites déraisonnables. On peut ici pointer le déficit d'information et d'explication concernant autant le droit et son évolution ainsi que le rapport de la société à la maladie mentale.

La tâche n'en est que plus ardue au moment de rendre compte de ce vote dans les textes. Le travail d'explication semble toujours en panne et le risque est grand que se prolonge dans l'application la même cécité qui a prévalu dans la campagne électorale quant au rôle du psychiatre dans la cité et au statut de la science dans cet éclairage difficile du comportement humain.

\section{Le «populisme pénal»: que peut signifier juger et punir en démocratie?}

On peut toujours se demander quelle fut la part de certaines officines politiques et des médias dans une telle décision, mais il n'en reste pas moins que le débat mené avant tout par les auteurs de l'initiative resta digne sans permettre qu'il se place là où il aurait dû être, à savoir au 
niveau du débat de société, puisque la plupart du temps, il a été présenté comme une affaire de spécialistes des prisons et des délinquants.

L'expression de D. Salas qui qualifie de «populisme pénal» les dérives actuelles des discours sur la sanction rend bien compte de ce qui est profondément en jeu dans nos sociétés modernes. Ces discours populistes, en prise directe avec la douleur des victimes, pointent l'insuffisance de la répression, l'incapacité des juges et insistent sur la nécessité de mesures d'exception.

D. Salas pointe l'utilisation qui en est faite dans les discours politiques et la réponse du législateur: «La législation compulsive se développe dans une société où l'urgence façonne la règle juridique» [2].

Ainsi les textes de loi sont votés au décours d'événements particulièrement chargés émotionnellement. Les gouvernements sont pris par la tentation démagogique.

«A partir du moment où le destinataire de la décision n'est plus l'individu mais le public, celui-ci en devient le critère et la justification», écrit encore D. Salas. C'est bien ce à quoi nous sommes dorénavant confrontés: écrire un texte de loi qui répond à l'attente du public, qui vient se glisser dans les failles de l'édifice pénal et qui en ignore les fondements.

Une telle inscription ne peut se faire qu'au détriment du développement de vrais moyens pour mieux comprendre les actes de délinquances sexuelles et surtout pour mieux prévenir leurs récidives: médiation, amélioration du suivi, du traitement individualisé, adaptation des peines, développement de recherches sur l'évaluation de la dangerosité et sur la compréhension du psychisme des auteurs d'actes délinquants, etc. Pourtant cette réflexion et la mise en place du travail de réinsertion et de réhabilitation devra se faire puisque aucune société n'a les moyens d'une politique conduisant à un enfermement carcéral exponentiel.

\section{Le rapport du psychiatre à la cité: une relation paradoxale}

Alors que le vote est un symptôme clair d'une défiance envers les psychiatres, le résultat du vote donnerait plus de pouvoir aux psychiatres, puisque la décision reposera, à priori, sur deux expertises qui seraient finalement les éléments centraux de la décision.

Il s'agit donc d'un paradoxe: donner plus de pouvoir tout en accroissant la défiance, puisque la loi impliquerait la responsabilité du psychiatre qui pourrait émettre un pronostic erroné.

On va le voir ci-dessous, il n'en est rien. Le texte ne propose finalement que la destruction du rôle du psychiatre dans cette articulation et son asservissement à une mission simple qui n'a plus grand chose à voir avec son art.

\section{Le texte adopté: un bouleversement du rapport à la loi et à I'expertise psychiatrique}

Le texte adopté renverse brutalement la relation du psychiatre à la procédure pénale dans deux aspects fondamentaux:

- L'oubli de ce que représente l'expertise psychiatrique, en tant que mise en évidence d'une pathologie psychiatrique.

- Le déni de ce que la reconnaissance d'une pathologie peut avoir comme conséquence pour le sujet dans son rapport à sa responsabilité et dans la détermination d'une mesure thérapeutique en adéquation avec le trouble et la dangerosité qui y est référée.

L'expertise psychiatrique, on le sait, a pour mission première de déterminer la responsabilité pénale du sujet auteur de délits ou de crimes.

Dans le texte de l'initiative point d'évocation de la pathologie éventuelle du sujet.

Jusqu'à présent l'application de l'article 43 CPS était marginale, voire tombée en désuétude dans certains cantons.

Ce nouvel article de loi vient finalement comme paradigme d'un nouveau droit des sanctions: le sujet labellisé dangereux échappe au droit commun pour faire l'objet d'une mesure d'exception, sur la base d'une expertise psychiatrique, celle-ci ne se prononçant, au bout du compte, que dans un domaine où, jusqu'à présent elle ne s'aventurait, avec raison, qu'avec la plus grande réserve, à savoir la détermination du risque de récidive ...

D'une certaine manière, il vient redonner vigueur au vieux rêve de Lombroso: permettre d'écarter de la sanction pénale classique les individus marqués par une dangerosité constitutionnelle et ouvrir la voie à un nouvel avatar du positivisme. On le comprend bien, le droit pénal étant trop clément (la perpétuité telle qu'elle est inscrite dans la peine ne remplissant pas sa fonction), il revient au criminologue (dont le psychiatre n'est que l'instrument) de définir une voie radicale d'exclusion.

Finalement, le débat sur la responsabilité est escamoté pour n'être plus qu'une simple figure de style, puisque le point de césure dans ce nouveau texte sera celui de la dangerosité dans ses aspects les plus éloigné de la compétence psychiatrique.

On pourra objecter que la sollicitation du psychiatre dans la détermination de la dangero- 
sité existait déjà. Mais, jusqu'à présent, elle ne concernait l'expertise avant jugement que de manière complémentaire et ne se déployait véritablement que dans la démarche d'évaluation de l'exécution de la peine.

Rappelons que Rappart fondait la création de la psychiatrie actuelle sur sa capacité à distinguer les sujets irresponsables des autres et à proposer à ceux ci d'autres voies de prise en charge.

Le souverain a balayé ce débat et il y a fort à parier que malgré le caractère exceptionnel d'une telle mesure, elle ne pourra que rejaillir sur l'ensemble des rapports entre droit pénal et psychiatrie.

\section{Implications pour le psychiatre:} déserter l'espace ou faire avec?

Les impasses médicales, conséquences du texte de cette initiative, sont nombreuses. Elles ont pourtant été balayées par la majorité du groupe qui a eu pour mandat de traduire ce vœu populaire en catégorie juridique:

En reformulant l'hypothèse d'une structure psychique du crime (cf. "critères structurels, étroitement et durablement liés à la personnalité de l'auteur») en ne référant cette définition qu'à une lecture de l'instant du jugement et présupposant un savoir qui n'existe pas: «si en raison des caractéristiques particulières de sa personnalité l'auteur peut être qualifié de durablement non amendable, dans la mesure où la thérapie semble, à longue échéance, pratiquement vouée à l'échec» (art. 64 ${ }^{\text {ter }}$, en projet).

En réduisant l'individu à son acte et référant sa structure psychique à la liste des actes commis «le juge ordonne l'internement à vie si l'auteur a commis un assassinat, un meurtre, une lésion corporelle grave, un viol, un brigandage, une prise d'otage, un incendie ou un autre crime pour lequel il a porté ou voulu porter gravement atteinte à l'intégrité physique, psychique ou sexuelle d'autrui» (art. 64 ${ }^{\text {ter }}$, lettre a).

Du coup, le débat sur la dangerosité et sur la prise en charge des délinquants souffrant de troubles psychiques s'en trouve singulièrement modifié, par rapport à tout ce qui peut se discuter de liens entre dangerosité et trouble psychique, sur la manière dont on peut appréhender la dangerosité dans une démarche qui prend en considération les multiples facteurs autant biographiques que cliniques ou conjoncturels.

En effet, la mise en œuvre du texte postule que la dangerosité est la pathologie majeure de ces délinquants, qu'elle s'exprime par un acte criminel qui en est donc le symptôme central et l'expression de sa personnalité et de son psychisme. On en revient d'une certaine manière à la définition de la monomanie homicide du XIX ${ }^{\mathrm{e}}$ siècle.
Implicitement le texte contient les éléments suivants:

- Il définit des catégories «à vie» de la «dangerosité» et de la non amendabilité en les habillant d'un alibi médical qui ne repose pourtant sur aucune validation scientifique crédible.

- Il exclut tout doute ou incertitude dans la démarche de prédiction; alors que celle-ci ne peut qu'être corrélée à une approximation statistique porteuse en soit d'incertitude.

- Il exclut toute possibilité d'évolution individuelles du psychisme et dénie d'une certaine manière le travail d'accompagnement et de cheminement qui peut être effectué, notamment à la faveur d'un accompagnement thérapeutique.

Bien sûr, on objectera que celui-ci ne concerne que des catégories très marginales de criminels, mais il ne peut que profondément teinter toutes les évolutions à venir car le vote populaire lui confère une légitimité qui ne peut que balayer les objections scientifiques. On le voit, ce texte témoigne d'une profonde fracture entre les réalités du monde médical et les exigences idéales de la population et de la Justice. Le leurre continue d'ailleurs puisque les principales difficultés retenues dans l'application sont présentées, à tort, comme prioritairement juridiques. Les réalités cliniques et scientifiques n'ayant manifestement pas été entendues

Face à un tel texte La Société Suisse de Psychiatrie argumente sur les points suivants [3]:

- En demandant la création des bases scientifiques nécessaires à l'appréciation de la validité de certains instruments pronostics en améliorant la formation, la qualité des expertises et la création de standards de qualité reconnus.

- En soulignant le caractère irréaliste de nouvelle études standardisées permettant de disposer de nouvelles connaissances, en raison même de l'impossibilité de standardiser ces études.

- En pointant l'insuffisance du nombre des experts en Suisse.

On ne peut que remarquer à quel point les arguments sont malaisés à mettre en forme tant la puissance du discours et sa force simplificatrice laisse peu de place au doute.

La question qui se pose nous amène à nous demander de quelle manière le dialogue peut exister dans un tel contexte. Déserter ou cautionner, c'est toujours le dilemme, en sachant que le train est en marche et que l'histoire indique que les mesures extrêmes trouvent toujours preneur. 


\section{La société et les monstres}

Ce nouvel article de loi laisserait en outre penser que tout acte monstrueux est le fait d'un malade inaccessible à un traitement.

La plupart du temps, les personnes visées par cette initiative ne présentent pas de maladie au sens où la médecine pourrait le reconnaître. C'est d'ailleurs la raison pour laquelle ces criminels sont toujours reconnus largement responsables de leurs actes.

Si l'on évoque un traitement, on doit donc parler de démarche effectuée dans le discernement et l'acceptation de ce à quoi le délinquant devenu, alors patient, doit s'affronter: horreur enfouie au fond de lui-même et qui l'a conduit à méconnaître l'autre. Il s'agit avant tout d'un cheminement qui permet au délinquant une démarche de réhumanisation.

La criminalité n'a pas une seule cause, elle en a de multiples. En dehors du psychisme et à l'intérieur du psychisme.

De grands progrès ont été faits dans la compréhension de ce qui peut conduire à des actes aussi terribles [4], mais nous nous trouvons avec ces travaux dans une toute autre approche que ce qui est visé par l'initiative. Toute cette réflexion et le travail développé dans de multiples lieux s'en trouvent balayés, dans l'attente d'hypothétiques nouvelles connaissances. Proposition dont le caractère quelque peu hypocrite pourrait sembler inciter à la recherche, alors que c'est bien de clôture d'une espace qu'il s'agit. Il y a des gens à exclure et des procédures à créer pour les exclure.

Le juge attendrait donc maintenant un travail de certitude sur le thérapeutique dont on sait qu'il n'est en soi pas possible. Il se résoudra à ne plus demander qu'un travail de pronostic dont on sait qu'il est écrasé par l'acte.

\section{Conclusion: Entre deux leurres, quel sens à la sanction actuellement?}

L'initiative évoque des sujets inamendables, tandis que les tenants d'une exécution de peine humaniste considèrent, de manière aussi irréaliste, «tout condamné comme un être non fini et perfectible».

Interner à vie certain délinquants reconnus comme dangereux permet le retour du discours déterministe et positiviste sur le criminel s'opposant ainsi à la philosophie des lumières et le libre arbitre.

Tout ceci sort le criminel d'un droit «commun» pour le plonger dans un droit d'exception où la sanction sera déterminée, lourdement, non pas directement par l'appréciation pénale de l'acte mais sur le lien hypothétique qui peut exister entre son psychisme et cet acte.

Et ce glissement s'effectuera sur la base d'une évaluation psychiatrique, «dépénalisant» en quelque sorte l'individu pour le confiner dans un champ d'arbitraire. C'est là le plus inquiétant, car ce que nous ne réalisons, peut-être pas assez, c'est que ce champ n'est pas celui de la psychiatrie légale pas plus qu'il n'est celui de la criminologie. Mais il s'agit d'un champ où l'objet n'a pas d'outils de pensée et où le discours psychiatrique en ressort dévoyé à l'extrême pour n'être plus que coquille vide, métrique propre à laisser le champ libre à tous les illusionnistes du sécuritaire. Au delà d'une clinique, c'est d'un nouveau marché qu'il s'agit.

\section{Quelles conséquences?}

Applicable, pas applicable, la question reste posée. Dans ce domaine toutes les dérives sont possibles, bien malin celui qui pourrait anticiper les conséquences. Mais il est certain que le système fonctionne déjà dans la tête des gens et rendra encore plus difficile le développement d'alternatives thérapeutiques, puisque la sécurité est maintenant garantie!

De tels textes ne posent plus la question de l'articulation nécessaire entre la psychiatrie et la loi à propos de l'expertise de la personne dont la folie imprègne l'acte.

Dorénavant, la psychiatrie est la loi pour ceux dont l'acte est folie par son horreur. Mais la psychiatrie qui se construit ainsi n'a plus grand chose à voir avec la médecine, c'est là aussi que se situe le leurre.

Il s'agira, au mieux d'une activité actuarielle reposant sur des échelles d'appréciations inspirées de certaines que l'on connaît (VRAG ou PCL) et référant l'individu à un calcul de probabilité. Au pire l'expertise se réduira à une péroraison morale, car qui pourra oser ne pas pronostiquer une récidive?

La seule alternative n'est elle pas dans l'insistance de notre profession à occuper ce domaine et à l'articuler avec les hommes de loi...?

\section{Références}

1 Vallotton A. L'initiative populaire pour une vraie perpétuité: les méfaits du sentiment d'insécurité en démocratie directe, mai 2004, http://Champpenal.revues.org

2 Salas D. Gare au populisme pénal. Libération, 24 juin 2005.

3 Prise de position Société Suisse de Psychiatrie du 15 avril 2005.

4 Balier C. La violence en Abyme. PUF, 2005 